Network Working Group

Request for Comments: 346

$\mathrm{NIC}: 10425$
Jon Postel

Computer Science

$\mathrm{UCLA}-\mathrm{NMC}$

30 May 72

Categories : Echo Plex, Satellite

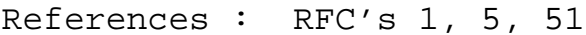

\title{
Satellite Considerations
}

The consideration of using space satellite transmission links in the ARPANET should be cause for some thought by the parties making use of the network. The satellite transmission path will not necessarily affect the transmission rate but it will affect the delay. The change in the delay characteristics can be approximated by the change in path length. Thus if the satellite is in synchronous orbit about 22,000 miles above the earth, the path length is about 44,000 miles compared to (worst case) 3,000 miles or about a 15 to 1 increase in path length and delay. (The time for light to travel 3,000 miles is .016 seconds, to travel 44,000 miles is .236 seconds.) In the current (surface) ARPANET delays are such that interactive servers with character-at-a-time remote echo are only marginally useful. While I believe that this delay (unmeasured) is largely due to the host systems, adding a half second transmission delay will cause these marginal systems to become unusable.

Thought should also be given to buffer allocations. If a receiving system allows only one line of text to be buffered at a time and refreshes the allocation as each line is output to a human user there will be at least a half second delay between the arrival of each line at the receiving system. This need not be a problem until the speed of the output device is above about 150 characters/second. This "small buffer" problem can be expected to occur even with lower speed devices since host delays are estimated to be in the range 0.1 second to 1.0 second. I suggest that it is appropriate to resume a discussion of measures to circumvent the difficulties brought about by these large delay characteristics. Some areas of discussion could be: buffer sizes in servers and users, echo plex techniques, moving part of the input processing to the user system. If it is decided to move the echo plex functions to the user system, it would be wise to try for a "standard" package, thus reducing a $\mathrm{M}$ times $\mathrm{N}$ problem to a $\mathrm{M}$ plus $\mathrm{N}$ problem. Please dig out and read RFC's \#1 Crocker, \#5 Rulifson, \#51 Elie to see some previous thinking about this type of problem.

[ This RFC was put into machine readable form for entry ]

[ into the online RFC archives by BBN Corp. under the

[ direction of Alex McKenzie. 12/96 ] 